\title{
XLVII. Theory of the hall effect in a binary electrolyte
}

\section{F. G. Donnan M.A. Ph.D.}

To cite this article: F. G. Donnan M.A. Ph.D. (1898) XLVII. Theory of the hall effect in a binary electrolyte, Philosophical Magazine Series 5, 46:282, 465-472, DOI: 10.1080/14786449808621218

To link to this article: http://dx.doi.org/10.1080/14786449808621218

曲 Published online: 08 May 2009.

Submit your article to this journal $[\pi$

Џ Article views: 2

Q View related articles $\asymp$ 
A theoretical consideration suggested by the phenomena of diffusive convection may not be uninteresting.

As the water flows along the capillary, friction occurs and heat is produced. Ultimately the dissolved salt mingles completely with the water. Instead of allowing the flow to occur along the capillary the tap $\mathrm{E}$ or $\mathrm{H}$ might be closed. The dissolved salt would then ningle completely with the water without producing any flow, and the final state (assuming the apparatus is thermally insulated) is exactly the same as before.

Now the principle of the conservation of energy indicates that the heat produced in the capillary plus the heat developed by the mixing of the solution with water is a constant.

Does not this indicate that the heat produced on mixing a solution with water depends on how the mixing takes place? Is the matter connected with a sort of surface-tension existing in the spaces between a strong and weak solution?

XLVII. Theory of the IIall Effect in a Binary Electrolyte. By F. G. Donnan, M.A., Ph.D.*

TN 1883 Roiti $\dagger$ investigated the subject of a possible Hall 1 effect in electrolytic solutions, but failed to obtain any positive result. Recently, howerer, the question has been taken up by Bagarà $\ddagger$, who has obtained very considerable effects in aqueous solutions of zinc and copper sulphates. On the other hand, negative results have been obtained by Florio \$, and as both Bayard and Florio maintain the correctness of their experimental work, a polemic on the subject has arisen between them. In this condition of affairs it seemed worth while to examine what effect might be expected theoretically. With this purpose in view I made the calculation contained in the following pages. Subsequently I discovered that a similar theory had been given by Van Everdingen, jun.\|; but as I do not arrive at quite the same results, and have considered the subject somewhat more generally, it seemed to me not to be needless repetition to communicate this note.

The basis of the following calculation may be stated briefly as follows. The diagram shows the directions of the primary current and the magnetic field. The lines of flow of the primary current are supposed to be straight and the magnetic

* Communicated by the Physical Society : read June 24th, 189 e.

$\dagger$ Journ. de Physique, 1883.

f Comptes Rendus, vol. exxii. pp. $77-79$, and cxxiii, pp. 1270-1273 (1896).

\$ Nuovo Cimento, $[4]$ vol. iv. pp. 106-111 (1896).

il Metingen over het Verschijnsel van Hall en de Toename van den Weerstand in het Magnetisch Veld, p. 102 et seq. (Leiden, 1897). 
field everywhere uniform. The effect of the ponderomotive forces on the moving ions in the magnetic field is to urge both positive and negative ions in the positive direction of $z$. The moving ions will thus acquire component velocities in this direction, and these velocities being in general different for each sort of ion, the result is the separation of positive and negative charges, whereby a potential-gradient is set up in the direction of the $z$-axis which reduces the originally unequal velocities to equality. We thus obtain a flow of ionic matter in the positive $z$-direction. This produces in its turn a reverse osmotic gradient or concentration-fall both for dissociated and undissociated salt. The stationary state is finally attained when the net flux of ionic matter in the positive direction of $z$ is balanced by the flux of undissociated salt in the opposite direction.

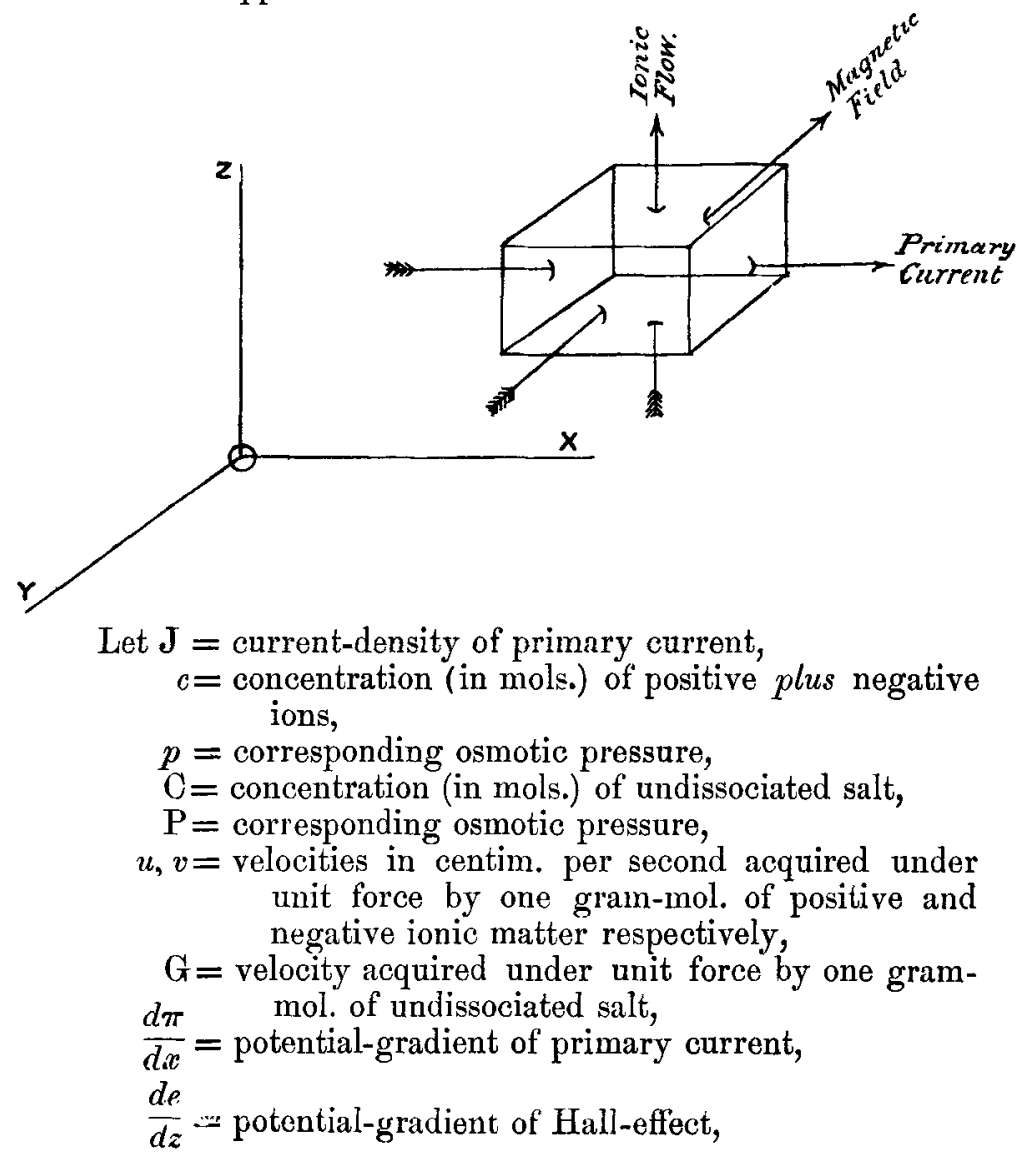


$\epsilon=$ quantity of electricity travelling per gram-equivalent of ionic matter,

$\omega=$ valency of each ion,

$\mathrm{H}=$ magnetic field-strength,

$t=$ temperature (absolute).

We shall suppose the laws $p=c \mathrm{R} t$ and $\mathrm{P}=\mathrm{CR} t$ to hold for the solution in question, although this is not essential. We have furthermore

$$
\mathrm{J}=-\frac{\omega^{2} \epsilon^{2} c}{2}(u+v) \frac{d \pi}{d x} . \quad . \quad . \quad . \quad .
$$

Now an ion with a positive charge $\omega e$ moving with a velocity $\mathrm{V}$ in the positive direction of axis of $x$ will be acted on by a force in the positive $z$ direction equal to $\omega \epsilon \mathrm{VH}$; so that the force in this direction on a positive gram-ion is given by $-\omega^{2} \epsilon^{2} u \mathrm{H} \frac{d \pi}{d x}$, whence it has a component velocity in the same direction amounting to $-\omega^{2} \epsilon^{2} u^{2} \mathrm{H} \frac{d \pi}{d x}$. By similar reasoning the velocity of a negative gram-ion in the positive $z$ direction is $-\omega^{2} \epsilon^{2} v^{2} \mathrm{H} \frac{d \pi}{d x}$.

Expressing quantities of matter in mols., we can now draw up the following list, which takes into account all the fluxes of matter occurring in the solution.

1. Quantity of positive ionic matter traversing per unit time unit section perpendicular to axis of $\approx$ in positive direction of this axis, due to ponderomotive forces arising from the magnetic field.

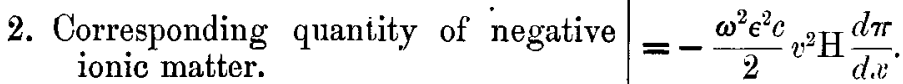

3. Flux of positive ionic matter in same direction due to potential-gradient along $z$-axis.

$=-\frac{\omega^{2} \epsilon^{2} c}{2} u^{2} \mathrm{H} \frac{d \pi}{d v}$

$=-\frac{\omega \epsilon c}{2} u \frac{d e}{d z}$

4. Corresponding flux for negative ionic
matter.

$\begin{aligned} & \text { 5. Flux of positive ionic matter in same } \\ & \text { direction due to osmotic gradient. }\end{aligned}=-u \frac{\mathrm{R} t}{2} \frac{d c}{d z}$.

$\begin{aligned} & \text { 6. Corresponding flux of negative ionic } \\ & \text { matter. }\end{aligned}=-v \frac{\mathrm{R} t d c}{2 d z}$.

$\begin{array}{r}\text { 7. Flux of undissociated salt in positive } \\ z \text { direction due to osmotic gradient. }\end{array}=-\mathrm{GR} t \frac{d \mathrm{C}}{d z}$. 
The stationary condition then gives the equations:-

$$
\begin{aligned}
& \frac{\omega^{2} \epsilon^{2} c}{2} u^{2} \mathrm{H} \frac{d \pi}{d x}+\frac{\omega \epsilon c}{2} u \frac{d e}{d z}+\frac{u \mathrm{R} t}{2} \frac{d c}{d z}+\mathrm{GR} \frac{d \mathrm{C}}{d z}=0, \\
& \frac{\omega^{2} \epsilon^{2} c}{2} v^{2} \mathrm{H} \frac{d \pi}{d x}-\frac{\omega \epsilon c}{2} v \frac{d e}{d z}+\frac{v \mathrm{R} t}{2} \frac{d c}{d z}+\mathrm{GR} t \frac{d \mathrm{C}}{d z}=0 .
\end{aligned}
$$

Elimination of $\frac{d \pi}{d x}$ and $\frac{d \mathrm{C}}{d z}$ from the equations (i.), (ii.), and (iii.) leads to the result

$$
\frac{d e}{d z}=\frac{2}{\omega \epsilon c} \frac{u-v}{u+v} \mathrm{HJ}-\frac{\mathrm{R} t}{\omega \epsilon c} \frac{u-v}{u+v} \cdot \frac{d c}{d z} . . . .
$$

It is to be observed that equation (iv.) holds for the variable as well as the stationary state, because although during the variable state the left-hand members of equations (ii.) and (iii.) are not zero, they are always equal to each other.

Accordingly for the initial phenomenon, before any appreciable concentration-gradient occurs, we obtain, putting $c=c_{0}$ and $\frac{d c}{d z}=0$,

$$
\frac{d e}{d z}=\frac{2}{\omega \epsilon c_{0}} \frac{u-v}{u+v} \mathrm{HJ}=\omega \epsilon(v-u) \mathrm{H} \frac{d \pi}{d x}, \quad . .
$$

or, integrating,

whence

$$
\iint \frac{d e}{d z} d y d z=\frac{2 \mathrm{H}}{\omega \epsilon c_{0}} \frac{u-v}{u+v} \iint \mathrm{J} d y d z
$$

$$
e=\frac{2}{\omega \epsilon c_{0}} \frac{u-v}{u+v} \frac{\mathrm{H} i}{d}, \quad . \quad . \quad . \quad . \quad . \quad . \quad . \quad .
$$

where $e=$ total difference of potential measured in direction of positive $z$-axis, $d=$ thickness measured in direction of magnetic field, and $i=$ primary current-strength. So that we obtain for the constant of the initial Hall-effect the value const. $=\frac{2}{\omega \epsilon c_{0}} \frac{u-v}{u+v}$.

In order to further investigate the stationary state, we shall suppose that the equilibrium-equation required by the laws of electroly tic dissociation is every where satisfied. 'This amounts to supposing that the processes which adjust this equilibrium proceed very much more rapidly than any of the other changes occurring in the system. In the uncertain state of knowledge concerning the equilibriam-equation it may be written for the 
present $\mathrm{C}=\phi(c)$; so that $\frac{d \mathrm{C}}{d z}=\phi^{\prime}(c) \frac{d c}{d z}$. Eliminating $\frac{d \mathrm{C}}{d z}$ between this equation and (ii.) and between this equation and (iii.), we obtain the equations

$$
\begin{aligned}
& \frac{d e}{d z}=\frac{2}{\omega \epsilon c} \frac{u}{u+v} \mathrm{HJ}-\frac{\mathrm{R} t}{\omega \epsilon c}\left\{\frac{2 \mathrm{G} \phi^{\prime}(c)}{u}+1\right\} \frac{d c}{d z}, . \\
& \frac{d e}{d z}=-\frac{2}{\omega \epsilon c} \frac{v}{u+v} \mathrm{HJ}+\frac{\mathrm{R} t}{\omega \epsilon c}\left\{\frac{2 \mathrm{G} \phi^{\prime}(c)}{v}+1\right\} \frac{d c}{d z} .
\end{aligned}
$$

Writing $\frac{2 G \phi^{\prime}(c)}{u}+1=\mathrm{L}, \frac{2 \mathrm{G} \phi^{\prime}(c)}{v}+1=\mathrm{M}$, we obtain from (vii.) and (viii.)

or

$$
\left.\begin{array}{l}
\frac{d e}{d z}=\frac{2}{\omega \epsilon} \frac{1}{\mathrm{~L}+\mathrm{M}} \frac{\mathrm{M} u-\mathrm{i} v}{u+v} \mathrm{HJ}, \\
\frac{d e}{d z}=\frac{\omega \epsilon}{\mathrm{L}+\mathrm{M}}(\mathrm{L} v-\mathrm{M} u) \mathrm{H} \frac{d \pi}{d x} .
\end{array}\right\} .
$$

Fór a completely dissociated electrolyte $\mathrm{L}=1, \mathrm{M}=1$, and we get instead of equations (ix.)

$$
\left.\frac{d e}{d z}=\frac{1}{\omega \epsilon c} \frac{u-v}{u+v} \mathrm{HJ}=\frac{\omega \epsilon}{2}(v-u) \mathrm{H} \frac{d \pi}{d x} . \quad \text { (ix. } a\right)
$$

Comparing equations (v.) and (ix, a), we see that the final potential-gradient for a completely dissociated electrolyte is just one-half the initial gradient.

Let $\frac{d e}{d z} / \frac{d \pi}{d x}=\mathrm{D}$, and denote by $\mathrm{U}, \mathrm{V}$ the velocities acquired under unit potential gradient by a gram-mol. of positive and negative ionic matter respectively. Equation (ix. $a$ ) may then be written

$$
\mathrm{D}=\frac{1}{2}(\mathrm{~V}-\mathrm{U}) \mathrm{H} . \quad \cdot \quad \cdot \quad \cdot \quad \cdot(\mathrm{x} .)
$$

Van Everdingen * arrived at the equation $\mathrm{D}=(\mathrm{V}-\mathrm{U}) \mathrm{H}$ for the stationary state in an electrolyte supposed to be very slightly dissociated; but be assumes in this case that the ionic concentration doos not vary throughout the solution, an assumption which is inconsistent with the equations (vii.) and (viii.), and therefore appears to me to be erroneous.

Equation ( $\mathrm{x}$.) may now be applied to the data obtained by Bagard. He finds, for example, the following results. 
$\mathrm{CuSO}_{4}$ solution. Concentration $=\frac{1}{16}$ grm. equiv. per litre. Temperature $21^{\circ}-26^{\circ} \mathrm{C}$.

\begin{tabular}{|c|c|c|c|}
\hline \multicolumn{2}{|c|}{ H. } & D. & $\frac{\mathrm{D}}{\overline{\mathrm{H}}}$. \\
\hline \multicolumn{2}{|c|}{385 C.G.S. units. } & .0018 & $46 \times 10^{-5}$ \\
\hline 707 & $"$ & $\cdot 0024$ & $\cdot 34 \times 10^{-5}$ \\
\hline 96 & $" \quad$, & $\cdot 0034$ & $35 \times 10^{-5}$ \\
\hline
\end{tabular}

To test these results by means of the theory, it is only necessary to calculate the value of $\frac{1}{2}(\mathrm{~V}-\mathrm{U})$, where it must be noticed that $\mathrm{V}$ and $\mathrm{U}$ are the velocities of the gram-mols. in centim. per second nnder a potential-gradient of one C.G.S. electromagnetic unit of potential per centim. From Ostwald's Lehrluch, vol. ii. p. 770, the molecular conductivity of $\mathrm{CuSO}_{4}$ solutions for the highest dilution at $18^{\circ} \mathrm{C}$. is $217^{\prime}$ in Siemens units, and therefore 230 in mho's. As the calculation is only very approximate, we may put consequently

$$
(\mathrm{U}+\mathrm{V})_{18^{\circ}}=\frac{230}{96540 \times 2 \times 10^{8}}
$$

From the same source, p. 612 , we get $\frac{U}{U+V}=\cdot 36$, a value which may be regarded as fairly correct for temperatures in the neighbourhood of $20^{\circ} \mathrm{C}$. (according to Hittorf and Bein, loc. cit.). Hence we obtain finally

$$
\frac{1}{2}(\mathrm{~V}-\mathrm{U})_{18^{\circ}}=16 \times 10^{-13} \text {. }
$$

The value of $\frac{1}{2}(\mathrm{~V}-\mathrm{U})$ for temperatures $21^{\circ}-26^{\circ} \mathrm{C}$. will probably be somewhat smaller. Of course the solution of concentration $=\frac{1}{16}$ grm.-equiv. per litre is not by any means completely dissociated; and by using the proper equation, namely,

$$
\overline{\mathrm{D}}=\omega \epsilon \frac{\mathrm{L} v-\mathrm{M} u}{\overline{\mathrm{L}}+\mathrm{M}}
$$

since $v>u$ and therefore $\frac{\mathrm{L} v-\mathrm{M} u}{\mathrm{~L}+\mathrm{M}}>\frac{v-u}{2}$, we should obtain a higher value for $\frac{D}{H}$. Nevertheless this would never account for the difference between $\cdot 39 \times 10^{-5}$ as observed by Bagard and $16 \times 10^{-13}$ as deduced theoretically for the case of complete dissuciation. 
So far as I can see, the theory here given is wholly in favour of the negative results obtained by Roiti and Florio; and it would therefore seem that Bagard has measured a phenomenon not contemplated in the foregoing theory. Van Everdingen in the paper referred to above supports Bagard; but this is owing to bis having accidentally omitted the factor $10^{-8}$ in his numerical work.

The ionic concentration-fall can be readily calculated for a completely dissociated electrolyte. From equations (iv.) and (ix. $a$ ) we get at once

$$
\mathrm{R} t \frac{d c}{d z}=\mathrm{HJ}
$$

or

$$
\frac{d \log c}{d z}=-\frac{\omega^{2} \epsilon^{2}}{2 \mathrm{R} t}(u+v) \mathrm{H} \frac{d \pi}{d x}
$$

whence

$$
\frac{1}{z_{2}-z_{1}} \log \frac{c_{1}}{c_{2}}=\frac{\omega \epsilon(\mathrm{U}+\mathrm{V})}{2 \mathrm{R} t} \mathrm{H} \frac{\pi_{2}-\pi_{1}}{x_{2}-u_{1}}
$$

or

$$
\frac{2 \mathrm{R} t}{\omega \epsilon} \log \frac{c_{1}}{c_{2}}=\mathrm{H}(\mathrm{U}+\mathrm{V})\left(z_{2}-z_{1}\right) \frac{\pi_{2}-\pi_{1}}{x_{2}-x_{1}} .
$$

Thus, calling $\mathrm{E}$ the P.D. between two transverse electrodes (of the same metal as the kation) due only to the differences in concentration set up (a case which could be realized by taking an electrolyte such as silver nitrate, for which $U$ and $\mathrm{V}$ are very nearly equal), it follows that

$$
\mathrm{E}=\frac{1}{2} \mathrm{H}(\mathrm{U}+\mathrm{V})\left(z_{2}-z_{1}\right) \frac{\pi_{2}-\pi_{1}}{x_{2}-x_{1}}
$$

Taking the case of copper sulphate, we get, as before,

$$
\mathrm{U}+\mathrm{V}=\cdot 00119 \text {. }
$$

Putting $z_{2}-z_{1}=1$ centim., and $\frac{\pi_{2}-\pi_{1}}{x_{2}-x_{1}}=1$ (volt per centim.), and $\mathrm{H}=20,000$ C.G.S. units, we obtain

$$
\mathrm{E}=12 \times 10^{-8} \text { volts. }
$$

Hence it would appear that in this, or in any other similar experimental arrangement, it would be necessary not only to employ an extremely strong magnetic field, but also a very high primary potential-gradient, $i$. e. of the order of 10,000 volts per centim. This might perhaps be realized experi- 
mentally by employing very dilute solutions and a large accumulator-battery, such as that recently employed by Professors Trowbridge and Richards*.

20 th May, 1898.

Note added May 27th.-Dr. Van Everdingen informs me that he has discovered the slip in his calculation, and agrees with the remarks made above concerning the experiments of Florio and Bagard.

XLVIII. On the Adtmissible Width of the Slit in Interference Experiments. By James W ALEeR, M.A. $\dagger$

1. TN a paper published in the Philosophical Magazine for 1 August 1889, Lord Rayleigh has discussed the question of the admissible width of the slit forming the proximate source of light in interference experiments, and has pointed out that "In Fresnel's experiment, whether carried out with mirrors or with biprism ... the condition for distinctness is simply that the width of the slit be a small proportion of the width of the bands $\neq . "$ This rule, which applies to the case in which the variation of the width of the bands is produced by a change in the mirrors or biprism, the distances of the slit and screen of observation from the apparatus remaining constant, requires modification when it is the variation of the distances that causes the alteration of the width of the bands, the apparatus remaining otherwise unchanged ; for it is an experimental fact that in this latter case, as the bands become finer, the slit may be made wider without loss of distinctness. A more detailed investigation leads to this result, and shows moreover that a progressive widening of the slit causes a periodic disappearance of the bands, the system of bands at the successive reappearances being alternately bright and dark centred $\S$.

2. We require first the relative retardation at a point of the screen of observation of the streams emanating from any point of the slit.

Fresnel's Mirrors.-Let the line of intersection of the mirrors be the axis of $y$, and let the axis of $z$ be taken so as to pass through the image of the centre of the slit in the

* Phil. Mag, February (1897).

† Communicated by the Author.

$\neq$ Phil. Mag. [5] xxviii. p. 80 (1889).

$\S$ I find that this result of the progressive widening of the slit has already been given by M. Ch. Fabry in his thesis for the degree of Doctor of Science, published at Marzeilles in 1892, and regret that I was not aware of this interesting work until my paper was in type. 